\title{
Disrupted cortical function underlies behavior dysfunction due to social isolation
}

\author{
Tomoyuki Miyazaki,,,2 Kenkichi Takase,,1,3 Waki Nakajima,, ${ }^{1}$ Hirobumi Tada, ${ }^{1}$ Daisuke Ohya, ${ }^{1}$ \\ Akane Sano, ${ }^{1}$ Takahisa Goto, ${ }^{2}$ Hajime Hirase, ${ }^{4}$ Roberto Malinow, ${ }^{5}$ and Takuya Takahashi1,6,7
}

\begin{abstract}
1Department of Physiology and 2Department of Anesthesiology, Yokohama City University Graduate School of Medicine, Yokohama, Japan. ${ }^{3}$ Department of Anatomy, Toho University School of Medicine, Tokyo, Japan. ${ }^{4}$ RIKEN BSI, Saitama, Japan. ${ }^{5}$ UCSD, La Jolla, California, USA. ${ }^{6}$ Albert Einstein College of Medicine, New York, New York, USA. " Japan Science and Technology Agency (JST),
\end{abstract} Core Research for Evolutional Science and Technology (CREST), Saitama, Japan.

\begin{abstract}
Stressful events during early childhood can have a profound lifelong influence on emotional and cognitive behaviors. However, the mechanisms by which stress affects neonatal brain circuit formation are poorly understood. Here, we show that neonatal social isolation disrupts molecular, cellular, and circuit developmental processes, leading to behavioral dysfunction. Neonatal isolation prevented long-term potentiation and experience-dependent synaptic trafficking of $\alpha$-amino-3-hydroxy-5-methylisoxazole-4-propionic acid (AMPA) receptors normally occurring during circuit formation in the rodent barrel cortex. This inhibition of AMPA receptor trafficking was mediated by an increase of the stress glucocorticoid hormone and was associated with reduced calcium/calmodulin-dependent protein kinase type II (CaMKII) signaling, resulting in attenuated whisker sensitivity at the cortex. These effects led to defects in whisker-dependent behavior in juvenile animals. These results indicate that neonatal social isolation alters neuronal plasticity mechanisms and perturbs the initial establishment of a normal cortical circuit, which potentially explains the long-lasting behavioral effects of neonatal stress.
\end{abstract}

\section{Introduction}

Mental stress can have different effects on the nervous system. While brief mild stress can evoke emotional arousal and enhance learning (1), chronic elevated stress can induce deficits in neuronal function $(2,3)$. In particular, social isolation early in life caused by neglect (a form of child abuse) can induce mental stress and lead to various mental illnesses such as depression, drug addiction, and anxiety disorders $(4,5)$. Because of the complex and poorly understood regulation of neuronal functions by stress $(6,7)$, elucidation of how stress affects the nervous system at the molecular, cellular, and circuit levels is an important step in controlling stress disorders.

Experience early in postnatal life is important to the establishment of normal functioning cortical circuits $(8,9)$. During this period, sensory experience through whiskers, an important form of social communication in rodents $(10,11)$, leads to a several-fold increase in excitatory synaptic transmission in the barrel cortex (12).

Excitatory synaptic transmission in the central nervous system is mainly mediated by $\alpha$-amino-3-hydroxy-5-methylisoxazole-4propionic acid-type (AMPA-type) glutamate receptors $(13,14)$. The number of AMPA receptors at synaptic sites determines the synaptic responses of postsynaptic neurons and controls neuronal activity (14-18). Stimuli inducing synaptic plasticity such as long-term potentiation (LTP) drive GluR1-containing AMPA receptors into synapses in vitro $(19,20)$. In vivo, whisker experience drives GluR1-containing AMPA receptors into synapses in the developing barrel cortex $(12,21,22)$. Synaptic addition of GluR1-containing AMPA receptors contributes to behavioral modification in several systems $(1,23-28)$.

A number of studies have revealed that behavioral stress, including social isolation, affects $\operatorname{LTP}(29,30)$. While some stress proto-

Conflict of interest: The authors have declared that no conflict of interest exists. Citation for this article: J Clin Invest. 2012;122(7):2690-2701. doi:10.1172/JCI63060. cols (e.g., inescapable restraint-tail shock, exposure to brightly lit unfamiliar chambers) attenuate LTP in the hippocampus (31-33), other protocols, such as brief exposure to a predator, enhance LTP (1). Therefore, the effect of stress on synaptic plasticity may be dependent on the stress protocol and show an inverted U-shape dose-response relation (relating the "dose" of stress and plasticity response). Recently, we have shown that brief arousal facilitates synaptic AMPA receptor delivery via norepinephrine-mediated (NE-mediated) signaling (1). However, the molecular mechanism of how behavioral stress attenuates synaptic plasticity is still unclear.

Rodents and humans may display similar effects of neonatal maltreatment on the nervous system (34-36). Here we examine the effects of early social isolation on synaptic function during cortical development. We focus on the synapses formed from layer 4 to layer $2 / 3$ in the developing rat barrel cortex (BC-L4, 2/3). We examine effects between P12 and P14, a time when GluR1-containing AMPA receptors are delivered into synapses by whisker experience and when the fine mapping in the barrel field is formed $(12,37)$. We find that brief periods of neonatal social isolation (but not merely whisker sensory deprivation) disrupt this process as well as LTP and result in the attenuation of whisker sensitivity at layer $2 / 3$ of the barrel cortex. This leads to disruption of whisker-dependent behavior. Furthermore, we find that these effects of neonatal social isolation are mediated by a glucocorticoid stress hormone. These results indicate that neonatal social isolation alters the formation of proper neuronal circuitry by a glucocorticoid signaling-mediated blockade of experience-dependent synaptic AMPA receptor insertion and leads to long-lasting behavioral perturbations.

\section{Results}

Social isolation disrupts whisker experience-dependent synaptic delivery of GluR1-containing AMPA receptors. In order to study the effect of social isolation on receptor trafficking, we isolated rat pups 
A

Postnatal day

7891011121314

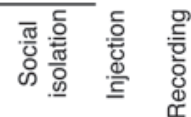
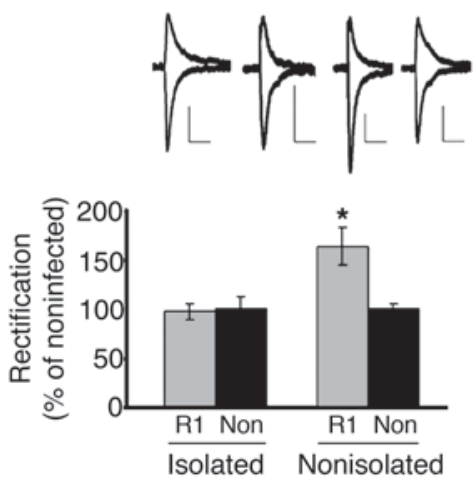

D

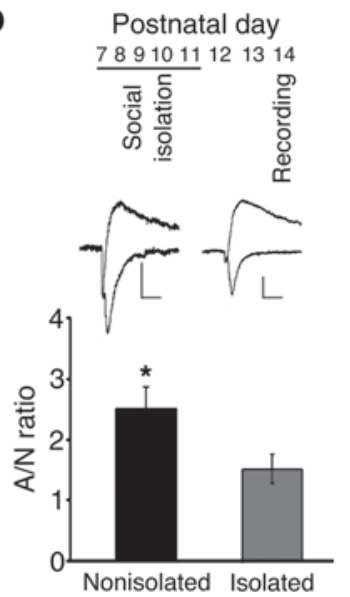

E
B
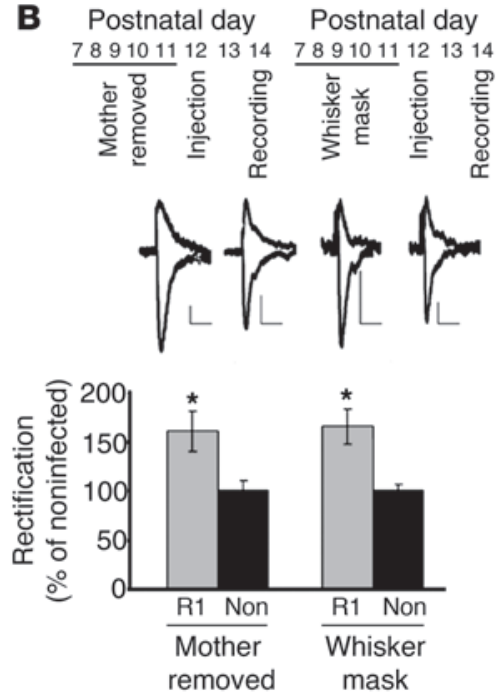

Postnatal day
7891011121314 7891011121314

Postnatal day

7891011 121314
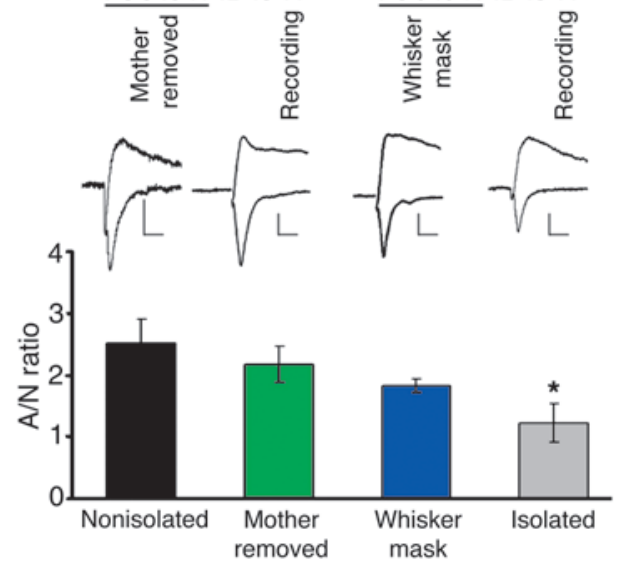

C Postnatal day 1234567891011121314

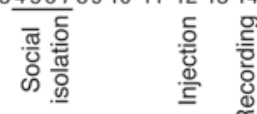
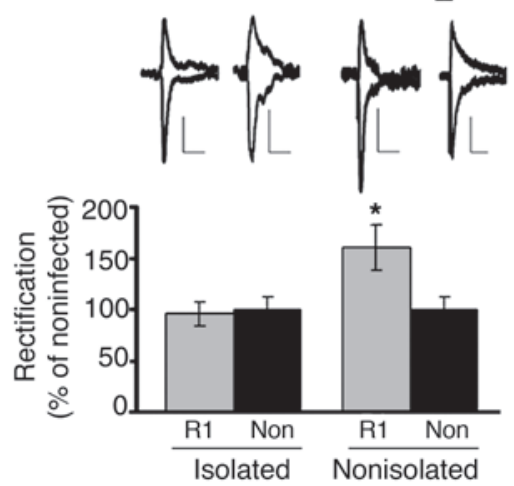

F

Postnatal day 4567891011121314

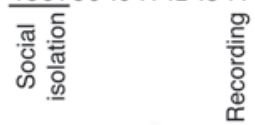

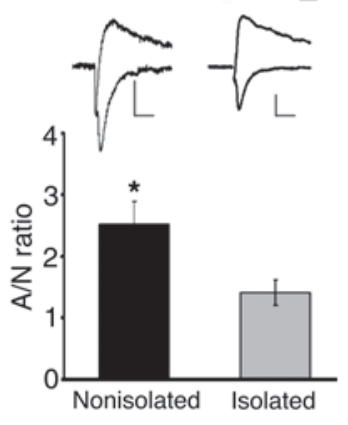

Figure 1

Social isolation disrupts experience-dependent synaptic delivery of AMPA receptors in the developing rat barrel cortex. (A-C) Top insets: time line of experiments. R1, GFP-GluR1-expressing neurons; non, nonexpressing neurons. (A) Isolation during P7-P11 disrupted GluR1 delivery at P12-P14 ( ${ }^{\star} P<0.05$, R1 versus non; $n=8$ nonisolated control, $n=9$ isolated). (B) Removal of mother during P7-P11 (mother removed) did not disrupt GluR1 delivery at P12-P14 ( ${ }^{*} P<0.05$, R1 versus non; $n=8$ ) (left). Blockade of whisker input during removal of mother (whisker mask) did not prevent GluR1 delivery ( ${ }^{*} P<0.05$, R1 versus non; $n=5$ ) (right). (C) Isolation (P4-P7) disrupted GluR1 delivery during P12-P14 $\left({ }^{\star} P<0.05\right.$, R1 versus non; $\left.n=6\right)$. (D) A/N ratio at P14 was lower with rats isolated than nonisolated rats $\left({ }^{\star} P<0.05\right.$, isolated vs, nonisolated; $n=18$ ). (E) A/N ratio at P14 of pups, either mother removed or whisker masked, was comparable with nonisolated rats, but larger than isolated animals $(n=16)\left({ }^{*} P<0.05\right.$, compared with nonisolated $[n=15]$, mother removed $[n=17]$, whisker mask $[n=21]$ animals; $\left.\mathrm{F}_{(3,65)}=4.022\right) .(\mathbf{F})$ $\mathrm{A} / \mathrm{N}$ ratio at $\mathrm{P} 14$ was lower with rats isolated at $\mathrm{P} 4-\mathrm{P} 7$ than nonisolated rats $\left({ }^{\star} P<0.05\right.$, isolated vs. nonisolated; $n=15$ nonisolated control animals, $n=18$ isolated animals). Scale bars: $20 \mathrm{pA} / 20 \mathrm{~ms}(\mathbf{A}, \mathbf{D}) ; 10 \mathrm{pA} / 20 \mathrm{~ms}(\mathbf{B}) ; 10 \mathrm{pA} / 15 \mathrm{~ms}$ (E, F). Data were analyzed by paired, 2 -tailed $t$ test $(\mathbf{A}-\mathbf{D}, \mathbf{F})$ or 1-way factorial ANOVA (E).

from their mothers and other siblings for 6 hours per day from P7 to P11 (38) (see Supplemental Chart of experimental procedures; supplemental material available online with this article; doi:10.1172/JCI63060DS1). At P12, we microinjected Sindbis virus expressing GFP-GluR1 in the barrel cortex of either isolated or nonisolated siblings from the same litter. We used low-titer virus to avoid toxic effects and infected about 1 of 1,000 cells in the injected area. Viral-mediated overexpression of GFP-GluR1 leads to about 4-fold increase in cell bodies and 35\% increase in dendrites of GluR1-containing AMPA receptors, indicating near physiological levels of AMPA receptor expression in infected den- drites (39). The expression level of GFP-GluR1 was comparable between isolated and nonisolated animals (data not shown). Furthermore, the basic electrophysiological properties such as input resistance and firing properties were indistinguishable between infected and noninfected neurons (Supplemental Table 1). These profiles were comparable between isolated and nonisolated animals (Supplemental Table 1). Recombinantly expressed GluR1 formed homomeric receptors which, in contrast with endogenous receptors, showed little outward current at positive potential, and thus synaptic incorporation of recombinant AMPA receptors was detected as an increased rectification (ratio of response at $-60 \mathrm{mV}$ 
A Isolated P7-P11, recorded P14

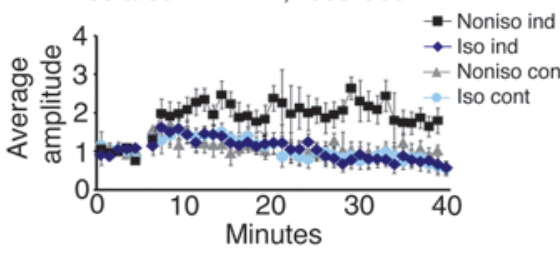

B Isolated P4-P7, recorded P14

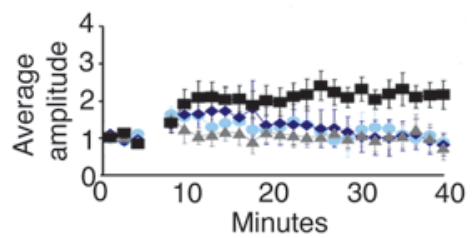

C Isolated P4-P7, recorded P27

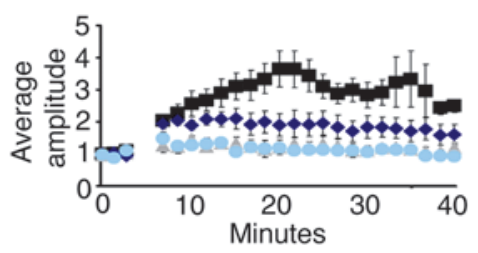

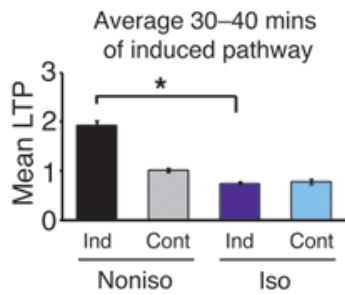
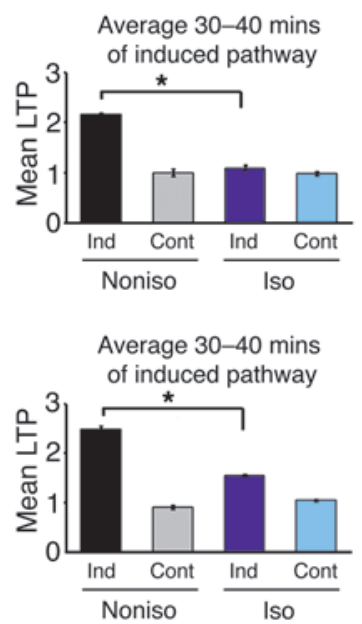

\section{Figure 2}

Social isolation disrupts LTP in the developing rat barrel cortex. LTP was induced by pairing $3-\mathrm{Hz}$ stimulation with depolarization of the postsynaptic neuron $(+20 \mathrm{mV})$ for 90 seconds. Recordings were maintained for at least 40 minutes after pairing. Mean amplitude between 30 minutes and 40 minutes after LTP induction was normalized to baseline amplitude (right). Social isolation during P7-P11 (A: ${ }^{*} P<0.05$, nonisolated induced pathway vs. isolated induced pathway; $n=6$ per each group) and P4-P7 (B: ${ }^{*} P<0.05$, nonisolated induced pathway vs. isolated induced pathway; $n=5$ per each group) prevented LTP at P14 in the barrel cortex. (C) Social isolation during P4-P7 partially blocked LTP in the barrel cortex at P27 $\left({ }^{\star} P<0.05\right.$, nonisolated induced pathway vs. isolated induced pathway; $n=6$ nonisolated control animals, $n=8$ isolated animals). Ind, LTP-inducing pathway; cont, control pathway; noniso, nonisolated animals; iso, isolated animals. Data were analyzed by paired, 2-tailed $t$ test. to $+40 \mathrm{mV}$ ) of AMPA receptor-mediated synaptic transmission. From P12 to P14, litters were maintained under normal conditions, and acute coronal brain slices were prepared at P14. Animals were maintained with whiskers intact throughout the experiment. We performed whole-cell recordings of layer 2/3-infected pyramidal neurons and nearby noninfected neurons and evoked transmission by stimulating layer 4. GFP-GluR1 infected neurons from nonisolated control siblings showed increased rectification compared with nearby noninfected neurons (Figure 1A). However, no increase of rectification was detected from GFP-GluR1-expressing neurons in isolated animals (Figure 1A). Therefore, experiencedriven synaptic delivery of GluR1 was blocked by social isolation at this early postnatal period. While isolation for 1 hour per day during P7-P11 or for 6 hours only at P7 still prevented experiencedriven synaptic GluR1 delivery during P12-P14, 1 hour isolation for just 1 day at P7 did not (Supplemental Figure 1A). Thus, the effect of social isolation on GluR1 delivery depends on the duration of isolation. In order to determine whether synaptic delivery of endogenous AMPA receptors was prevented by social isolation, we measured the ratio of AMPA to NMDA responses $(A / N)$. In brain slices from P14 animals isolated between P7 and P11 for 6 hours per day, the A/N ratio for transmission at BC-L4, 2/3 synapses was significantly lower than that observed in nonisolated animals of the same age (Figure 1D), indicating that social isolation from P7 to P11 disrupted synaptic delivery of endogenous AMPA receptors. The kinetics of NMDA receptor-mediated synaptic responses was not different between isolated and nonisolated animals, indicating that NMDA receptor-mediated synaptic currents are relatively stable (Supplemental Figure 1B). Consistent with these results, synaptosome fraction of isolated animals (6 hours per day during P7-P11 and assayed at P11) contained a reduced amount of GluR1 compared with that of nonisolated animals (Supplemental Figure 1C).
Since weaning occurs at a later stage, pups from P7 to P11 depend on nutrition provided by the dam. Therefore, pups were deprived of nutrition during social isolation. Although we did not detect any difference of body weights between isolated and nonisolated animals (Supplemental Table 2), we wished to rule out the possibility that the lack of nutrition during social isolation was responsible for the disruption of synaptic delivery of GluR1. We separated pups from the dam, but kept 10 pups together for 6 hours per day from P7 to P11 (mother removed). Thus, these pups were deprived of nutrition from the dam, but retained social interaction with other pups. In these pups, GFP-GluR1-infected neurons showed increased rectification, indicating synaptic incorporation of GFP-GluR1 (Figure $1 \mathrm{~B}$ ). The $\mathrm{A} / \mathrm{N}$ ratio for transmission at BC-L4, 2/3 synapses of pups deprived of the dam was not different from that of control pups, but was significantly larger than that of isolated animals (Figure 1E), indicating that mother removal from P7 to P11 did not disrupt synaptic delivery of endogenous AMPA receptors. This shows that experience-driven synaptic delivery of GluR1 in the development was intact in pups separated from the mother if they retained social interaction with other pups. Thus, disruptions in nursing do not appear responsible for the observed deficits.

During 6 hours per day of social isolation, pups did not interact with other siblings or the mother, and thus they may have had reduced whisker experience. A reduction of whisker experience during social isolation procedure could be responsible for the disruption of synaptic delivery of GluR1 by social isolation. To rule out this possibility, we deprived whisker experience for 6 hours per day from P7 to P11 by masking whiskers (whisker mask). This method is effective in depriving experience, as continuous masking for 2 days from P12 to P14 blocks experience-dependent delivery of GluR1 into BC-L4, 2/3 synapses (infected, $102 \% \pm 15 \%$; noninfected, $100 \% \pm 17 \% ; P>0.5)$. In order to prevent their mother 

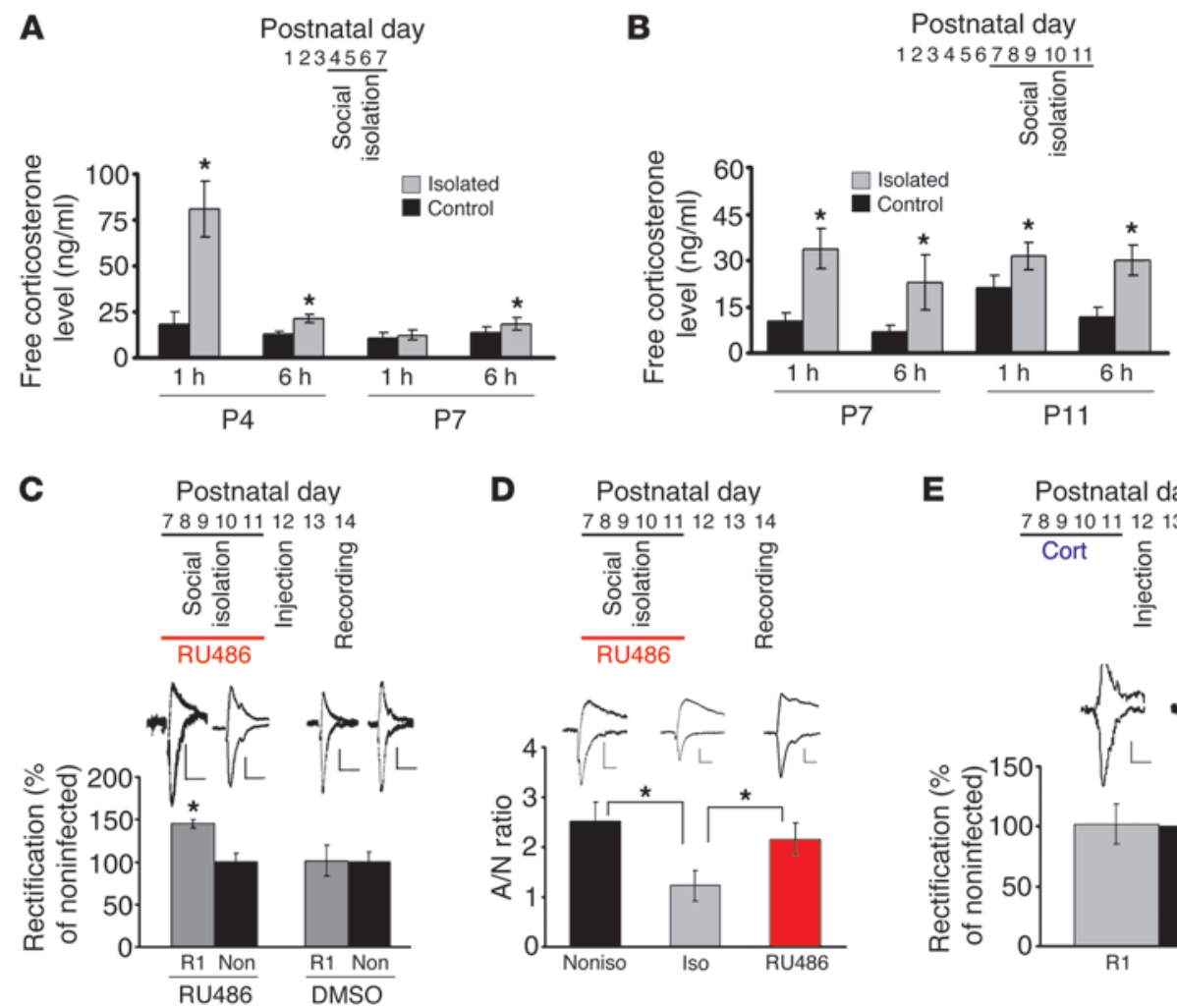
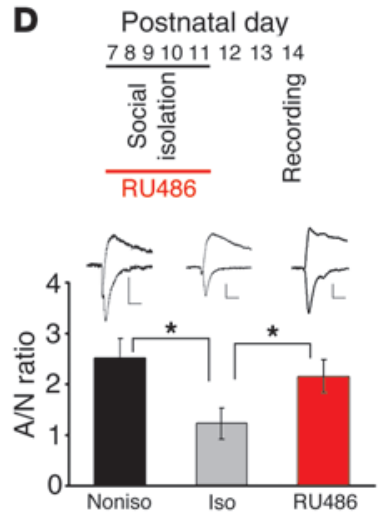

E
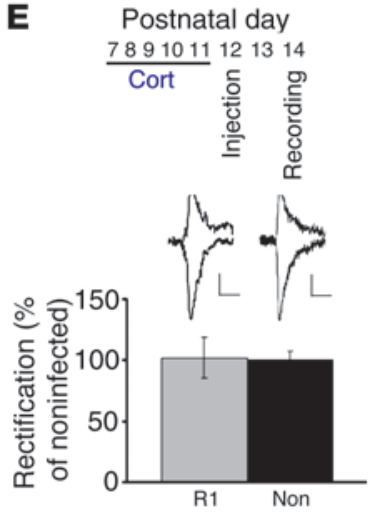
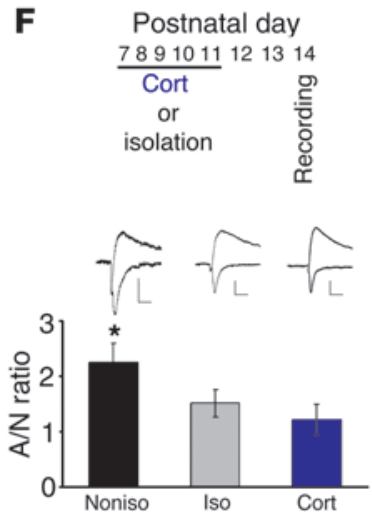

G Cort injection P7-P11, recorded P14
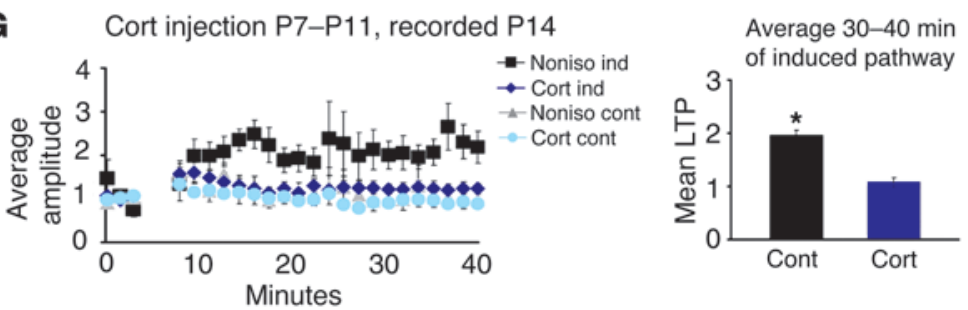

\section{Figure 3}

Increased glucocorticoid mediates synaptic plasticity disruption by isolation. (A and $\mathbf{B}$ ) Isolation increased free corticosterone levels from blood samples collected at 1 hour and 6 hours after the initiation of isolation $\left({ }^{*} P<0.05\right.$ compared with control animals, A: isolated at P4-P7, assayed at P4 and P7, B: isolated at P7-P11, assayed at P7 and P11; $n=10)$. (C) Administration of RU486 prevented the disruption of GluR1 delivery by isolation at P7-P11 ( ${ }^{*} P<0.05, \mathrm{R} 1$ versus non; $\left.n=5\right)$, while vehicle showed no effects $(n=5)$. (D) A/N ratio at P14 of isolated pups with RU486 was comparable with nonisolated, but larger than isolated animals without $R U 486\left(\mathrm{~F}_{[2,41]}=3.481,{ }^{\star} P<0.05\right.$, isolated vs. nonisolated, isolated versus RU486; $n=15$ ). (E) Administration of corticosterone (Cort) disrupted synaptic GluR1 delivery of animals housed in a normal environment $(n=8)$. (F) A/N ratio at P14 of nonisolated pups treated with corticosterone $(n=12)$ was lower than nonisolated $\left(\mathrm{F}_{(2,35)}=3.395\right.$, ${ }^{*} P<0.05$, nonisolated vs. isolated, nonisolated vs. Cort, $n=15)$, but comparable with isolated rats $(n=16)$. (G) Attenuated LTP at P14 of animals treated with corticosterone ( ${ }^{\star} P<0.05$, control vs. corticosterone-injected animals; $n=7$ nonisolated control animals, $n=8$ nonisolated animals with corticosterone). Ind, LTP-inducing pathway; cont, control pathway. Scale bars: 10 pA/20 ms (C, E); 10 pA/15 ms (D, F). Data were analyzed by paired, 2-tailed $t$ test $(\mathbf{A}-\mathbf{C}, \mathbf{E}, \mathbf{G})$ or 1-way factorial ANOVA (D, F).

from removing the mask, we separated a masked animal from the dam, but kept it with several other siblings to retain social interaction with other pups. We expressed GFP-GluR1 in the barrel cortex from P12 to P14 in these masked animals. We found that GFPGluR1-expressing cells showed increased rectification (Figure 1B). The $\mathrm{A} / \mathrm{N}$ ratio at these synapses of pups with masks was not different from that of control pups, but was significantly larger than that in isolated animals (Figure 1E), indicating that whisker masking for 6 hours per day from P7 to P11 did not disrupt synaptic delivery of endogenous AMPA receptors. This indicates that deprivation of whisker experience for 6 hours per day from P7 to P11 did not have any effect on experience-driven synaptic incorporation of GluR1 from P12 to P14.

Taken together, social isolation from P7 to P11 disrupts whisker experience-dependent synaptic delivery of GluR1 at P12 to P14. This effect is neither due to lack of nutrition from the dam nor possible reduction of sensory experience during social isolation.

Social isolation at early postnatal stage disrupts experience-driven synaptic delivery of GluR1 later in development. We examined longer-term effects of social isolation on experience-driven synaptic deliv- 
A

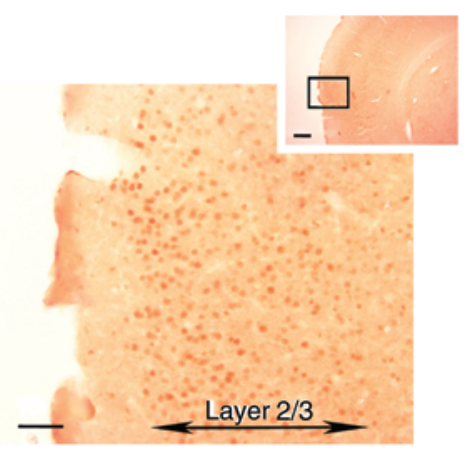

D

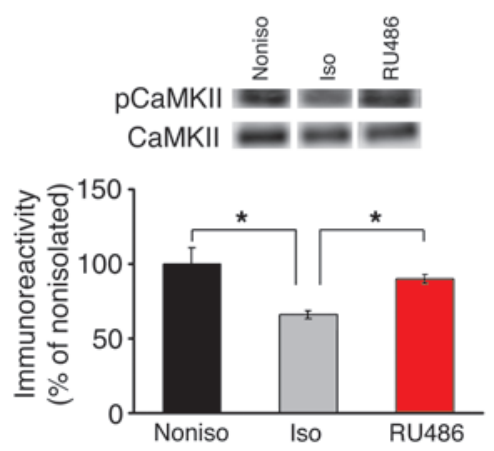

B

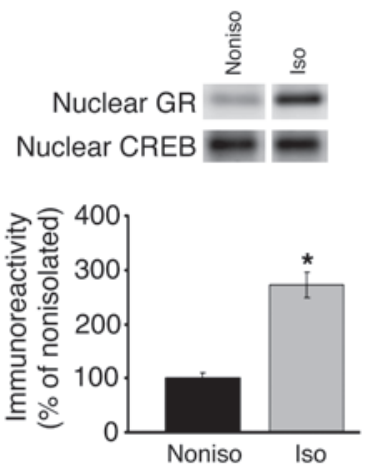

E

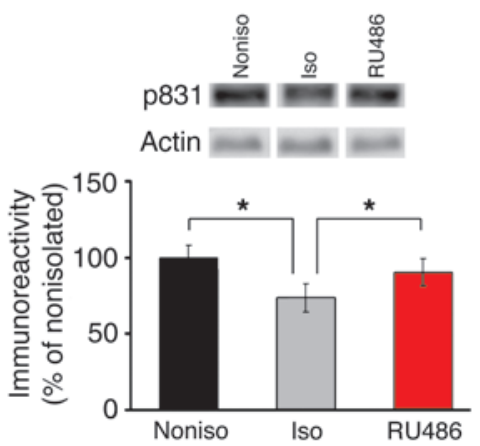

C
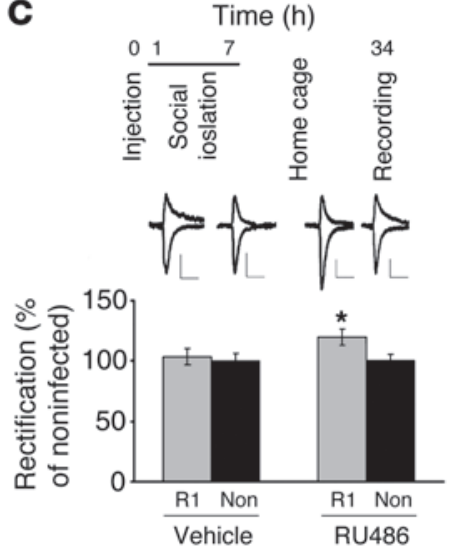

F 78910111213

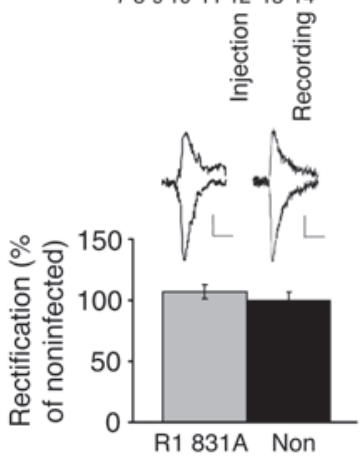

Figure 4

Mechanisms underlying isolation-induced disruption of synaptic AMPA receptor delivery. (A) Immunohistostaining of the barrel cortex of animals at P11 with anti-GR antibody. (B) Nuclear fraction contained larger amount of GR at 3 hours after social isolation than nonisolated animals. The amount of GR in the nuclear fraction of isolated animals was normalized to nonisolated rats $\left({ }^{*} P<0.05\right.$, isolated vs. nonisolated; $n=6$ per each group). (C) Local injection of RU486 during social isolation prevented the disruption of synaptic GluR1 delivery by social isolation. Top inset showed time line of experimental manipulations (see text) ( ${ }^{\star} P<0.05$, R1 versus non; $n=9$ isolated with RU486, $n=7$ isolated with vehicle). (D) Social isolation decreased phosphorylation of CaMKII at Thr286 compared with nonisolated rats. Phosphorylation level (calculated as the ratio to total CaMKII) of rats with social isolation in the presence of RU486 was comparable to nonisolated rats $\left(\mathrm{F}_{(2,11)}=8.163\right.$, ${ }^{*} P<0.05 ; n=5$ per each group). (E) Social isolation decreased phosphorylation of Ser831 of GluR1 compared with nonisolated rats. Phosphorylation level of rats with social isolation in the presence of RU486 was comparable to nonisolated rats but larger than isolated animals without $R U 486\left(F_{(2,45)}=6.950\right.$, ${ }^{*} P<0.05 ; n=12$ per each group). (F) No delivery of 831A (R1 831A) at layer 4-2/3 synapses in the barrel cortex of intact rats at P12-P14 ( $\left.n=16\right)$. Scale bars: $200 \mu \mathrm{m}(\mathbf{A}) ; 100 \mu \mathrm{m}$ (A, inset); 10 pA/20 ms. (B, D, and E); $20 \mathrm{pA} / 20 \mathrm{~ms}$ (C). Lanes were run on the same gel but are noncontiguous. Data were analyzed by paired, 2-tailed $t$ test $(\mathbf{B}, \mathbf{C}, \mathbf{F})$ or 1-way factorial ANOVA (D, E).

ery of AMPA receptors. Pups were isolated from the mother and other siblings for 6 hours per day from P4 to P7 and then maintained under normal conditions from P8 to P14; GFP-GluR1 was expressed from P12 to P14 by viral-mediated gene transfer. Wholecell recording of slices prepared at $\mathrm{P} 14$ showed no increase of rectification of GFP-GluR1-expressing cells, while normal GFP-GluR1 delivery into synapses was observed in nonisolated control siblings (Figure $1 \mathrm{C}$ ). Consistent with this, the $\mathrm{A} / \mathrm{N}$ ratio for transmission at BC-L4, 2/3 synapses of isolated animals was significantly lower than that observed in nonisolated animals of the same age (Figure 1F). This indicates that social deprivation early in development disturbs synaptic delivery of GluR1 1 week later in development.

Social isolation disrupts LTP. Above, we show that social isolation disrupts experience-dependent delivery of AMPA receptors in vivo. This could be due to a disruption of biochemical pathways necessary for the trafficking of AMPA receptors. Since similar biochemical pathways are used between LTP and experience-driven synaptic delivery of AMPA receptors $(14,16,17,19)$, disturbance of molecular machinery for the delivery of AMPA receptors should result in the disruption of LTP. Alternatively, rats exposed to social isolation could develop aberrant social behavior that could lead to inappropriate sensory stimuli (e.g., reduction of contact through whiskers) during the period when receptor trafficking requires whisker activity; this aberrant experience could prevent synaptic delivery of AMPA receptors. In this case, neurons of isolated rats could retain normal endogenous biochemical pathways that would permit LTP in vitro. In order to distinguish these 2 possibilities, we examined the effect of social isolation on LTP tested in vitro.

Acute slices were prepared from P14 rats that had been isolated either from P4 to P7 or from P7 to P11 for 6 hours per day. Wholecell recordings were obtained from layer $2 / 3$ pyramidal neurons, 
A
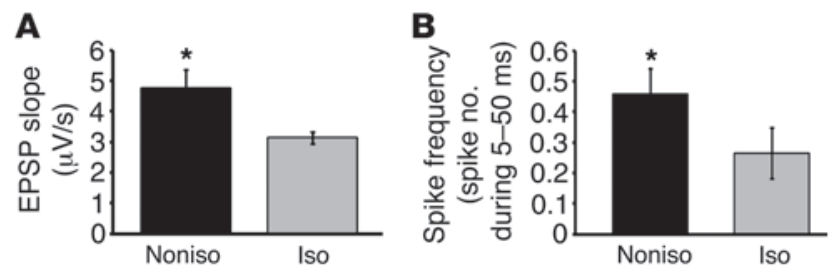

C
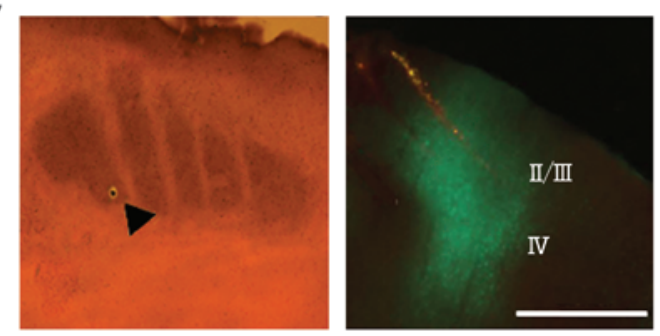

D

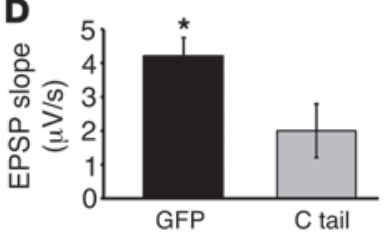

\section{Figure 5}

Social isolation attenuates the sensitivity of whiskers. Responses (field EPSP slopes and the frequency of spikes) in layer $2 / 3$ neurons of the barrel column to the deflection of principal whiskers. (A and $\mathbf{B}$ ) The sensitivity of whiskers was attenuated by social isolation. (A) Rats with social isolation exhibited lower-field EPSP slopes than control animals, indicating attenuated sensitivity of whiskers $\left({ }^{*} P<0.05\right.$, nonisolated versus isolated; $n=10$ per each group). (B) Rats with social isolation exhibited lower spike frequency (spike number during 5-50 ms from whisker stimulation) than control animals, indicating decreased sensitivity of whiskers $\left({ }^{*} P<0.05\right.$, nonisolated versus isolated; $n=10$ per each group). (C) Wire electrode was implanted into almost identical tangential location of barrel column (arrowhead indicates the location of the implanted electrode) throughout experiments (left). Example of implanted electrode into the layer $2 / 3$ of the barrel cortex (right). Coronal cortical slices infected with GFP-GluR1ct-expressing virus. The electrode was implanted into layer $2 / 3$ of the barrel cortex (layer numbers are indicated). Scale bar: $500 \mu \mathrm{m}$. (D) Rats with GFP-GluR1ct (C tail) expression exhibited lower-field EPSP slopes than GFPexpressing animals, indicating decreased sensitivity of whiskers by the expression of GFP-GluR1ct ( ${ }^{*} P<0.05$, GFP versus $C$ tail; $C$ tail, $n=7$, GFP, $n=4$ ). Data were analyzed by paired, 2-tailed $t$ test. and transmission was evoked by stimulating layer 4. LTP was induced by pairing synaptic stimulation with postsynaptic depolarization ( $3 \mathrm{~Hz},+20 \mathrm{mV}, 90$ seconds). While nonisolated rats showed intact LTP, paired-induced LTP was significantly reduced in siblings exposed to social isolation (Figure 2, A and B). We examined the effect of early social isolation on LTP at a later developmental age (P27). Although we did not detect attenuation of LTP induced by the same protocol as used in young animals in isolated rats (data not shown), social isolation during $\mathrm{P} 4-\mathrm{P} 7$ partially attenuated LTP induced by the weaker condition $(1 \mathrm{~Hz}, 0 \mathrm{mV}, 90$ seconds), indicating that the milder but persistent effect of neonatal social isolation continues until juvenile age (Figure 2C). These data indicate that biochemical pathways necessary for the trafficking of AMPA receptors were perturbed by this social isolation protocol.

Glucocorticoid mediates social isolation-induced disruption of AMPA receptor delivery into synapses. What mediates the disruption of experience-driven synaptic delivery of AMPA receptors by social isolation? Given that plasticity was globally affected, we considered corticosterone, a major stress hormone in rodents. Since the active form of corticosterone is free corticosterone (rather than that bound to corticosterone-binding globulin), we measured the free blood corticosterone level (see Methods). During social isolation experiments, prolonged rise of free blood corticosterone levels was observed (Figure 3, A and B).

Next, we injected RU486, an antagonist of glucocorticoid receptor (GR), during 6 hours per day social isolation from P7 to P11. As described above, GFP-GluR1-expressing Sindbis virus was injected into layer $2 / 3$ of the barrel cortex at P12, and acute brain slices were prepared at $\mathrm{P} 14$. Whole-cell recording of slices prepared from isolated rats injected with RU486 (8 $\mu \mathrm{g} / \mathrm{g}$ of body weight) revealed increased rectification of infected neurons compared with noninfected neurons (but there was no statistical difference between the rectification of infected neurons from isolated animals with RU486 and those from control nonisolated animals; control infected, $138 \% \pm 7 \%$; noninfected, $100 \% \pm 3 \%, n=27, P<0.05$; isolation with RU486 infected, $143 \% \pm 12 \%$; noninfected, $100 \% \pm 5 \%$, $n=7, P<0.05$, no statistical difference between "control infected" and "isolation with RU486 infected", $P>0.5$ ), indicating normal rescue of synaptic delivery of GluR1 (Figure 3C and Supplemental Figure 2A). Injection of vehicle (DMSO) did not block the effect of social isolation in both cases (Figure $3 \mathrm{C}$ ). The $\mathrm{A} / \mathrm{N}$ ratio for transmission at BC-L4, 2/3 synapses of pups isolated for 6 hours per day in the presence of RU486 (8 $\mu \mathrm{g} / \mathrm{g}$ of body weight) was not different from that of control nonisolated pups, but was significantly larger than that of isolated animals (Figure 3D), indicating that blockade of GR with RU486 during social isolation rescued isolation-induced disruption of synaptic delivery of endogenous AMPA receptors. The effect of RU486 was dose dependent, since isolated animals injected with reduced RU486 $(1.6 \mu \mathrm{g} / \mathrm{g}$ of body weight) were not rescued (Supplemental Figure 2A).

In order to elucidate further the role of increased glucocorticoid on experience-dependent synaptic delivery of AMPA receptors, we determined whether injection of glucocorticoid mimics the effect of social isolation. Animals injected with corticosterone (4.17 $\mu \mathrm{g}$ per day) from P7 to P11 were housed in a normal environment (see Supplemental Chart). Then a GFP-GluR1-expressing virus was injected into rat barrel cortex at P12, and acute slices were prepared at P14. Whole-cell recording showed no difference of rectification between GFP-GluR1-expressing and nonexpressing neurons, indicating no synaptic delivery of GFP-GluR1 (Figure $3 \mathrm{E}$ ). Furthermore, the $\mathrm{A} / \mathrm{N}$ ratio for transmission at $\mathrm{BC}-\mathrm{L} 4$, $2 / 3$ synapses of nonisolated animals injected with corticosterone was significantly lower than that observed in nonisolated animals without injection and comparable to that of isolated pups, indicating that endogenous AMPA receptor delivery was prevented by the injection of corticosterone (Figure $3 \mathrm{~F}$ ). Consistent with these results, injection of this amount of corticosterone attenuated LTP at P14 (Figure 3G). Injection of reduced corticosterone 


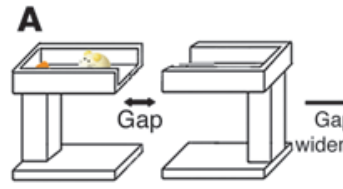

Can cross

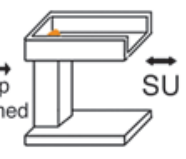

Rat stops crossing

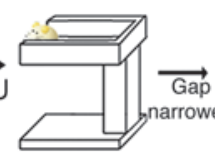

$\Delta \mathrm{S}(\mathrm{mm})=\mathrm{SU}-\mathrm{SL}$

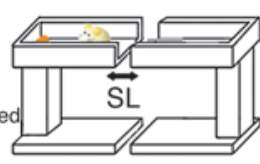

Rat starts crossing again
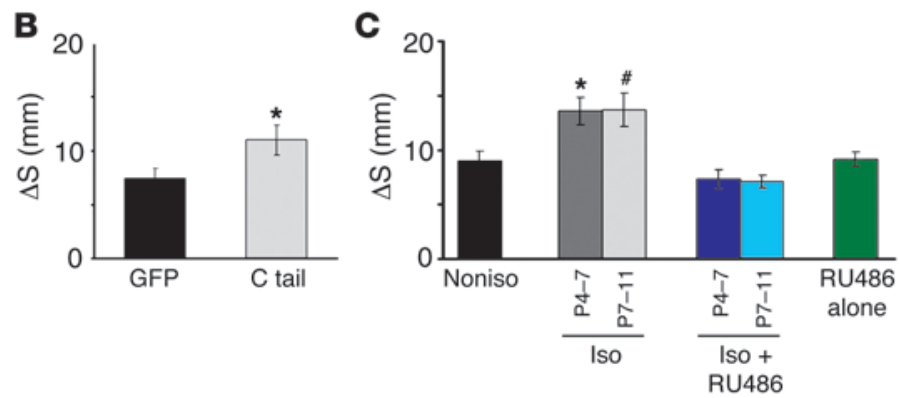

\section{Figure 6}

Social isolation disrupts behavior dependent on whisker-barrel function. (A) Schematic of gap-crossing test. The gap was widened until rats could no longer cross (middle, SU), and then the gap was narrowed until they could cross (right: SL). Difference between SU and SL is indicated by $\Delta S$ (see text and Methods for details). (B) Rats with GFP expression in the barrel cortex showed lower $\Delta$ S than GFP-GluR1ctexpressing ( $C$ tail) animals, indicating that GFP-expressing animals have better distance detection ability than GFP-GluR1ct-expressing animals $\left({ }^{*} P<0.05\right.$, GFP versus $C$ tail; $n=8$ per each group). (C) Isolated rats showed higher $\Delta S$ than nonisolated rats, indicating worse distance detection. Isolated rats treated with RU486 showed lower $\Delta S$ than untreated isolated rats, indicating RU486 rescued distance detection of isolated rats $\left(\mathrm{F}_{(5,45)}=8.668,{ }^{*} P<0.05\right.$ compared with nonisolated $[n=9]$ and isolated postnatal P4-P7 with RU486 [ $n=9]$ animals, ${ }^{\#} P<0.05 \mathrm{com}$ pared with nonisolated and isolated P7-P11 with RU486 [ $n=8]$ animals). RU486 treatment alone had no effect on distance detection $(n=8)$. Data were analyzed by paired, 2-tailed $t$ test (B) or 1-way factorial ANOVA (C).

(0.83 $\mu \mathrm{g}$ per day) did not block GFP-GluR1 delivery, showing a dose-dependent effect of corticosterone (Supplemental Figure 2B). These results indicate that elevated glucocorticoid levels are sufficient to prevent experience-driven AMPA receptor trafficking to synapses, as is seen with social isolation.

To determine whether social isolation activates glucocorticoid signaling at the barrel cortex and prevents experience-dependent synaptic AMPA receptor delivery, we first examined whether social isolation induces nuclear translocation of GR in the barrel cortex, a mechanism for activation of glucocorticoid signaling. Immunostaining with anti-GR antibody exhibited the expression of GR in the layer $2 / 3$ neurons of the barrel cortex at P11 (Figure $4 \mathrm{~A})$. Three hours of social isolation at P11 increased the amount of GR in the nuclear fraction (Figure 4B). These data indicate that social isolation activates glucocorticoid signaling at the barrel cortex. If social isolation-induced prevention of AMPA receptor delivery is mediated by the activation of GR in the barrel cortex, local injection of RU486 into the barrel cortex should block the effect of social isolation. To test this, we injected RU486 together with virus expressing GFP-GluR1 at P12 (see Methods). One hour later, we isolated rat pups for 6 hours. Pups were returned to the home cage, and whole-cell recordings were performed 27 hours later (Figure 4C). GFP-GluR1-expressing neurons showed increased rectification in the presence of RU486, while

no increase of rectification from infected neurons was detected in the absence of RU486, indicating that local injection of RU486 prevented the effect of social isolation (Figure 4C). We found no statistical difference in the rectification index (RI) between infected neurons from control nonisolated animals (measured 34 hours after virus injection without social isolation) and those from isolated animals with RU486 (control animals: infected, $131 \% \pm 9 \%$; noninfected, $100 \% \pm 7 \%$, $n=9$; isolated animals with RU486: infected, $120 \pm 7 \%$, noninfected, $100 \% \pm 5 \%, n=8, P>0.2$ between infected neurons from control animals and isolated animals with RU486), indicating normal rescue of synaptic delivery of GluR1. These results suggest that blockade of synaptic AMPA receptor delivery with social isolation in the barrel cortex was mediated by the local activation of glucocorticoid signaling.

Social isolation decreases CaMKII activity and phosphorylation of GluR1 at a site critical for its synaptic delivery. Activation of calcium/calmodulin-dependent protein kinase type II (CaMKII) is critical for synaptic AMPA receptor delivery, LTP, and behavioral plasticity (40). To elucidate the signaling mechanisms that mediate the connection between social isolation and synaptic GluR1 insertion, we first examined the effect of social isolation on CaMKII activity. Synaptosome fractions from the barrel cortex of isolated animals ( 6 hours per day during P7-P11) with or without RU486 and nonisolated animals were prepared at P11. Then phosphorylation of Thr286 of CaMKII $\alpha$, an indicator of CaMKII activation, was assessed. We found that social isolation decreased phosphorylation at Thr286, indicating downregulation of CaMKII activity (Figure 4D). This effect of social isolation was prevented in the presence of RU486 (8 $\mu \mathrm{g} / \mathrm{g}$ of body weight) (Figure 4D). Downregulation of CaMKII activity by neonatal social isolation persisted until a later developmental age (P27) (nonisolated, $100 \% \pm 15 \%$; isolated, $61 \% \pm 10 \%, n=12, P<0.05$; data not illustrated), consistent with the attenuation of LTP at P27 by early social isolation (Figure 2C).

Ser831 of GluR1 is a target of CaMKII and known to be phosphorylated during LTP (41). We next examined the effect of social isolation on phosphorylation at Ser831 of GluR1. Synaptosome fraction from the barrel cortex of isolated animals exhibited decreased phosphorylation at Ser831 compared with that of nonisolated control animals (Figure 4E). Phosphorylation levels at Ser831 of rats with social isolation in the presence of RU486 ( $8 \mu \mathrm{g} / \mathrm{g}$ of body weight) were comparable to those of nonisolated control rats, but significantly larger than those of isolated animals without RU486 (Figure 4E). Consistent with the persistent downregulation of CaMKII activity until juvenile age in isolated animals, we found that phosphorylation of Ser831 was still reduced at P27 in isolated animals (nonisolated, $100 \% \pm 5 \%$; isolated, $69 \% \pm 9 \%, n=12, P<0.05$ ). We did not observe the change of the phosphorylation at Ser845 with social isolation (data not shown).

Previous studies indicate that mutation of GluR1 at Ser831 to alanine does not prevent its synaptic incorporation in neurons expressing constitutively active CaMKII, indicating that increase of phosphorylation at Ser831 was not required for this form of plasticity (19). To determine whether this is the case with experi- 
ence-dependent synaptic delivery of GluR1 in vivo, we expressed GluR1 mutant (Ser831 to alanine: 831A) in the barrel cortex at P12 and examined whisker experience-driven delivery of $831 \mathrm{~A}$ at layer 2/3-4 synapses. We detected no difference of rectification between $831 \mathrm{~A}$-expressing and nonexpressing neurons at P14, indicating no synaptic delivery of 831A (Figure 4F). Thus, phosphorylation of Ser831 is required for experience-dependent synaptic GluR1 delivery. Expression of constitutively active CaMKII may activate mechanisms other than those produced by input from sensory experience in vivo and could overcome lack of phosphorylation at Ser831.

These results suggested that social isolation downregulates CaMKII activity via glucocorticoid, decreases phosphorylation at Ser831, and results in attenuation of synaptic GluR1 delivery.

$N E$ does not mediate social isolation-induced disruption of synaptic AMPA receptor delivery. NE is also a crucial stress hormone in mammals. In order to elucidate the role of $\mathrm{NE}$ in the effect of social isolation, we injected propranolol $(3.0 \mathrm{mg} / \mathrm{kg})$, a blocker of $\beta$-adrenergic receptors, during social isolation from P7 to P11. We used this dose of propranolol, since we found that increase of phosphorylation at Ser845 of GluR1 induced by the systemic injection of epinephrine $(0.5 \mathrm{mg} / \mathrm{kg}$ i.p.) was blocked in the presence of propranolol (3.0 mg/kg i.p.), suggesting the effectiveness of propranolol at this dose (data not shown). As was described above, synaptic delivery of recombinant GFP-GluR1 was assessed by whole-cell recording. Isolated animals showed no difference of rectification between GFP-GluR1-expressing and nonexpressing neurons even in the presence of propranolol (Supplemental Figure 3A), indicating no synaptic delivery of GFP-GluR1 receptors. Synaptic delivery of endogenous AMPA receptors was also prevented by social isolation in the presence of propranolol, since the $\mathrm{A} / \mathrm{N}$ ratio of isolated rats with propranolol at P14 was significantly lower than that of nonisolated rats and comparable to that of isolated animals without propranolol (Supplemental Figure $3 \mathrm{~B})$. These results indicate that social isolation-induced disruption of experience-dependent synaptic delivery of AMPA receptors in the developing rat barrel cortex requires glucocorticoid signaling, but not NE signaling.

Whisker-barrel function is attenuated by social isolation. Disruption of AMPA receptor trafficking to BC-L4, 2/3 synapses could lead to an aberrant functional relationship between whiskers and layer $2 / 3$ barrel columns. Since the effect of neonatal social isolation during P4-P7 on LTP persisted until juvenile age (Figure 2C), we chose juvenile age to assess the effect of early isolation (P4-P7) on whisker-barrel function.

The decrease of synaptic transmission of BC-L4, 2/3 synapses by the disruption of synaptic GluR1 delivery could result in an attenuation of the sensitivity of each barrel to the corresponding whiskers. To examine this, we measured the slope of field excitatory postsynaptic potential (EPSP) and the discharge frequency of individual neurons in response to the deflection of whiskers with in vivo tetrode recordings from a single barrel. Whiskers with the highest response were defined as principal whiskers. We collected data from recording electrodes placed in layer $2 / 3$ of the barrel field and at the almost identical tangential location within the barrel column (Figure 5C). Single-unit activities were semiautomatically isolated based on the spike waveform using the ND Manager software suite (ref. 42 and Supplemental Figure 4, A-C). As we excluded fast-spiking interneurons from the analysis, the majority $(>90 \%)$ of the isolated unit activities originated from pyramidal cells, from which we detected synaptic dysfunction (see above).
First, we examined the response to principal whisker deflections of rats either with or without social isolation. Rats were either isolated for 6 hours per day from P4 to P7 or kept under normal conditions. We measured the slope of field EPSP and the frequency of spikes in response to the deflection of principal whiskers at P23. We found that the slope of field EPSP and the discharge frequency by the deflection of principal whiskers of isolated animals were significantly smaller than those of control animals, indicating that the sensitivity of the barrel to its principal whisker was attenuated (Figure 5, A and B). There was no difference in spike latency by the deflection of the principal whisker between animals with social isolation during P4-P7 and control nonisolated rats at P23 (Supplemental Figure 4D). This suggests that social isolation deteriorates the sensitivity of the barrel column to its principal whisker at layer $2 / 3$. Absolute values of spike frequency and field EPSP at layer 4 of the barrel cortex from isolated animals displayed no difference with those of nonisolated control rats (Supplemental Figure 4, E and F), suggesting that the decrease of the response to the whisker deflection at layer $2 / 3$ was not inherited from dysfunction at layer 4.

Next, we wished to relate the attenuation of whisker-barrel function to the disruption of experience-dependent synaptic delivery of GluR1. We expressed the cytoplasmic portion of GluR1 tagged with GFP (GFP-GluR1ct) in layer $2 / 3$ of the barrel cortex, beginning at P4, using lentivirus-mediated in vivo gene transfer. GFPGluR1ct prevents experience-driven synaptic delivery of endogenous GluR1 $(12,23)$. We found that the slope of field EPSP in response to a deflection of the principal whisker was significantly smaller in GFP-GluR1ct-expressing rats than in control GFPexpressing rats, indicating that the sensitivity of the barrel to its principal whisker was attenuated in GFP-GluR1ct-expressing rats (Figure 5D). These results suggest that social isolation attenuates the sensitivity of the barrel column to its principal whisker by the disruption of GluR1 delivery to BC-L4, 2/3 synapses.

Social isolation attenuates whisker-dependent behavior. We next examined the effects of the attenuated whisker sensitivity on behavior. We assessed the disruption of whisker sensitivity with the ability of an animal to detect distances using a modified version of the gap-crossing task (see Methods), which is known to be dependent on the detection ability of whiskers (refs. 43, 44, and Figure 6A). In this task, rats were initially trained to find a reward at the opposite end of a runway. After this training phase, a gap was inserted in the runway and was widened at $0.5-\mathrm{cm}$ intervals. The gap was widened until rats would no longer step across it. This gap size was defined as the upper threshold (SU). Subsequently, the gap was narrowed at $0.5-\mathrm{cm}$ intervals until rats crossed it (Figure 6A). This gap size was defined as the lower threshold (SL). The difference between SU and SL was calculated (defined as $\Delta \mathrm{S}$ ). Rats touched the target platform even on noncrossing trials (data not shown), suggesting that rats utilize their whiskers to perform this task.

Since the effect of neonatal social isolation on synaptic plasticity and whisker-barrel function lasts until juvenile age (Figure 2C and Figure 5), we performed whisker-barrel function-dependent behavior task at a juvenile age.

We first used this test on P21 animals that had been expressing either GFP-GluR1ct or GFP in layer $2 / 3$ of the barrel cortex (delivered at P4 by lentivirus-mediated in vivo gene transfer). GFPGluR1ct-expressing animals showed larger distance discrimination values $(\Delta S)$ than GFP-expressing animals, indicating that expression of GFP-GluR1ct impaired the distance-detection abil- 
ity (Figure 6B). Therefore, this gap-crossing test is dependent on AMPA receptor trafficking and proper whisker-barrel function.

Next, animals were exposed to social isolation for 6 hours per day either during P4-P7 or P7-P11. At P21, rats were tested in the gap-crossing task. Figure $6 \mathrm{C}$ shows distance discrimination values $(\Delta S)$ of rats housed in control nonisolated environments and of isolated rats treated with RU486 $(8.0 \mu \mathrm{g} / \mathrm{g}$ of body weight $)$ or without RU486 during social isolation. $\Delta \mathrm{S}$ was significantly different among the groups. Post hoc analyses revealed that control rats showed lower $\Delta \mathrm{S}$ than rats with social isolation, indicating a better distance detection ability in control rats (Figure 6C). Moreover, isolated rats treated with RU486 showed lower $\Delta \mathrm{S}$ than isolated rats without RU486, but no difference was observed between control and RU486-treated isolated rats, indicating that RU486 rescued the effect of social isolation on the ability of distance discrimination (Figure 6C). Application of RU486 did not affect this behavior task, since nonisolated rats treated with RU486 exhibited the same level of distance discrimination ability as control animals (Figure 6C). It is unlikely that these effects of social isolation on this task were due to general anxiety, since we observed no difference of indicators of anxiety on the open field apparatus and the device used for the gap-crossing task between isolated and nonisolated rats (Supplemental Figure 5, A and B). Moreover, there was no significant difference in food consumption, suggesting no effect of social isolation on the motivation in this task (Supplemental Figure 5C). Furthermore, we did not detect any difference in the length of whiskers between isolated and nonisolated animals (isolated, $22.4 \pm 0.4 \mathrm{~mm}$; nonisolated, $23.3 \pm 0.7 \mathrm{~mm}, n=7, P>0.3$, D2 whisker), suggesting that this stress protocol did not have any effect on whisker growth. These results indicate that neonatal social isolation impaired the distance discrimination ability of animals and that this effect was mediated by the glucocorticoid pathway.

Since social isolation during P4-P7 induced synaptic defect at early developmental age (P14), we determined whether disruption of whisker-dependent behavior appeared at P14. To test this, we used the forelimb-placing test (45), which can be used on animals of this age. In this test, a pup's body was supported by the experimenter so that the limbs of the pup were free. Then, whiskers were brushed. If barrel function is normal, a pup extends the forelimb contralateral to deflected whiskers, but not if it has aberrant barrel function. We counted the number of normal limb responses per 10 trials. We found significantly lower scores of normal forelimb response in animals isolated either during P4-P7 or P7-P11 than in pups housed under normal conditions (Supplemental Figure 6). This indicates that impairments in whisker-dependent behavior also occurred at the same age at which synaptic dysfunction was detected.

\section{Discussion}

While numerous studies have revealed profound effects of stress on neural function, few have comprehensively related molecular, synaptic, and circuit deficits to behavioral aberrations $(30,46,47)$. In this study we found that the stress produced by neonatal social isolation disrupts synaptic delivery of AMPA receptors, circuit formation in the cortex, and behaviors related to somatosensory function. We focused on plasticity in the barrel cortex, since it is likely to play an important role in the integration of social information. This region is the primary sensory cortical area receiving input from whiskers, which are important for social interaction $(10,11)$. Although we analyzed only the barrel cortex in this study, we elucidated the molecular mechanism underlying the effect of neonatal isolation on synaptic plasticity, which may play an important role in the pathophysiology of many CNS diseases.

We examined the potential signaling mechanisms underlying the effects of isolation. We found that a prolonged rise of corticosterone level was induced by neonatal social isolation. This downregulates CaMKII activity and phosphorylation at Ser831 of GluR1, and this could contribute to the interruption of experience-driven synaptic delivery of AMPA receptors, while other posttranslational modifications with social isolation could be involved in the disruption of synaptic AMPA receptor delivery. NE, another important stress hormone, does not mediate the effect of social isolation on AMPA receptor trafficking, since propranolol did not prevent social isolation-induced disruption of AMPA receptor delivery. A recent in vitro study showed that brief treatment with corticosterone increases synaptic AMPA receptors (48). In contrast with the effects of chronic elevation of corticosterone observed with social isolation, a lower brief rise of glucocorticoid might enhance synaptic delivery of AMPA receptors, suggesting an inverted $U$ dose-response effect of glucocorticoid signaling on AMPA receptor trafficking. By what molecular mechanism glucocoritcoid signaling alters CaMKII activity remains to be elucidated. One possible mechanism is an increase in a protein phosphatase such as calcineurin and attenuation of CaMKII activity through dephosphorylation of phosphorylated active CaMKII. Indeed, chronic stress was shown to increase the calcineurin level in neurons (49). Downregulation of CaMKII activity in isolated animals persisted until juvenile age (P27), when attenuation of LTP was still observed in isolated animals, suggesting that neonatal chronic stress could induce permanent changes in signaling machinery required for synaptic plasticity. Although PKC could also be involved in the phosphorylation of Ser831, social isolation did not decrease PKC activity, suggesting that decrease in the phosphorylation of Ser831 was not due to the downregulation of PKC activity (data not shown). Social isolation induced the reduction of CaMKII activity as well as the decrease of the phosphorylation of Ser831 on GluR1. Social isolation-induced decrease of the phosphorylation of Ser831 could be due to the reduction of CaMKII activity or to mechanisms other than CaMKII action. Thus, our results delineate molecular mechanism underlying long-lasting effects of neonatal chronic stress on synaptic function.

Sensory processing is crucial for cognitive functions such as attention, emotion, memory, and social behavior. Alterations in sensory processing constitute prominent symptoms in various psychiatric disorders (e.g., autism, schizophrenia, and personality disorders) (50-53). While genetic alterations are considered to be important as etiologies of these mental illnesses, postnatal environment could also contribute to the onset of these mental disorders. In human studies, it has been shown that traumatic events such as child abuse could lead to misprocessing of sensory information (54-58). Our findings show that neonatal social isolation, which could be a traumatic experience, can cause disruption of experience-dependent synaptic delivery of AMPA receptors and aberrant circuit formation in the rodent somatosensory cortex and leads to behavioral abnormalities. Since rodents and humans may display similar effects of neonatal maltreatment on the nervous system (36), elucidation of molecular and cellular events in the somatosensory cortex of animals with postnatal maltreatment could lead to the discovery of novel biomarkers of mental disorders induced by postnatal severe stress such as child abuse. 


\section{Methods}

Animals. Subjects were Sprague-Dawley (SD) rats (Charles River Laboratories), and multiple colonies that contained 5 males and 5 females were used. Rats were housed in plastic ekon cages and maintained on a 14-hour light/10-hour dark cycle (full light at 0500 and full darkness at 1900). Temperature was held constant at $22^{\circ} \mathrm{C} \pm 1{ }^{\circ} \mathrm{C}$ and humidity at $55 \% \pm 5 \%$. Food and water were provided ad libitum.

Postnatal manipulations. For the social isolation experiment, 2 male pups were isolated from mother and other siblings for 6 hours per day from P4 to P7 or P7 to P11 from 1,000 hours to 1,600 hours (see Supplemental Chart of experimental procedures). Each isolated male pup was placed in a smaller separate cage (so that each stayed alone in a cage), which was kept on a heat pad at $35^{\circ} \mathrm{C}$ in an adjacent room during the isolation period. All pups were separated as a group from the dam for 6 hours per day from P4 to P7 or P7 to P11 from 1,000 hours to 1,600 hours. Separated pups were kept in a new cage, which was kept on a heat pad at $35^{\circ} \mathrm{C}$ in an adjacent room during the removal period. Whiskers of male pups were covered with Scotch tape (3M) for the same period as described above. Two male pups with whiskers masked were kept with 8 other pups together, but separated from the dam as above.

Infection of neocortical neurons in vivo. Constructs of GluR1 and GluR1ct tagged with GFP and Sindbis viruses were prepared as previously described $(19,59,60)$. Viral-mediated in vivo gene transfer and whisker deprivation were performed as previously described (12). Briefly, at P12, rats were anesthetized with a ketamine/xylazine cocktail (ketamine, $0.56 \mathrm{mg} / \mathrm{g}$ body weight; xylazine, $0.03 \mathrm{mg} / \mathrm{g}$ body weight) and positioned in a stereotaxic apparatus. Injections of viral solutions were delivered at $200 \mathrm{nl}$ with a glass micropipette through a skull window by pressure application (10-15 psi). The injections were performed within the following stereotaxic coordinates: $-2.0 \mathrm{~mm}$ from bregma; $4.5 \mathrm{~mm}$ lateral from midline, and $0.5 \mathrm{~mm}$ vertical from cortical surface. Subsequently the skin was repositioned and maintained with cyanacrylate glue. GFP-tagged GluR1ct was amplified by PCR and subcloned into FUGW vector with EcoR1 and BamHI sites. Then, lentivirus carrying GFP-GluR1ct was produced as previously described (61). Lentivirus-expressing GFP-GluR1ct or GFP was injected into layer 2/3 of entire barrel cortex at P4. Functional whisker-barrel map and behavior of injected animals were investigated as described below.

Electrophysiology. Two days after virus injection, rats were anesthetized with isoflurane gas, and acute brain slices were prepared and whole-cell recordings were performed as previously described (12). For rectification experiments, we added $0.1 \mathrm{mM}$ DL-APV to the perfusate to block NMDA receptors. There were no significant differences in input or series resistance among experimental groups (Supplemental Table 1). Rectification index (RI) was calculated as the ratio of AMPA receptor-mediated responses at $-60 \mathrm{mV}$ to responses at $+40 \mathrm{mV}$ (over $50-100$ responses were averaged). $\mathrm{RI}$ values of infected neurons were always normalized to those of uninfected neurons. A/ $\mathrm{N}$ ratios were calculated as the ratio of peak current at $-60 \mathrm{mV}$ to the current at $+40 \mathrm{mV} 50 \mathrm{~ms}$ after stimulus onset (40-50 traces averaged for each holding potential). LTP was induced by pairing $3-\mathrm{Hz}$ stimulation with depolarization of the postsynaptic neuron to $+20 \mathrm{mV}$ (isolated during P4-P7 or P7-P11 and recorded at P14 from the barrel cortex) or $1 \mathrm{~Hz}$ with $0 \mathrm{mV}$ (isolated during $\mathrm{P} 4-\mathrm{P} 7$ and recorded at P27 from the barrel cortex) for 90 seconds; recordings were maintained for at least 40 minutes after pairing. The excitatory postsynaptic current (EPSC) amplitude throughout recordings was always normalized to the average baseline amplitude before pairing. Bath solution contained $119 \mathrm{mM} \mathrm{NaCl}, 2.5 \mathrm{mM} \mathrm{KCl}, 4 \mathrm{mM} \mathrm{CaCl}$, $4 \mathrm{mM} \mathrm{MgCl}_{2}, 26 \mathrm{mM} \mathrm{NaHCO}_{3}, 1 \mathrm{mM} \mathrm{NaH}_{2} \mathrm{PO}_{4}, 11 \mathrm{mM}$ glucose, and 0.1 $\mathrm{mM}$ picrotoxin and were gassed with $5 \% \mathrm{CO}_{2}$ and $95 \% \mathrm{O}_{2}$. Patch-recording pipettes (3-5 MOhm) were filled with internal solution, $\mathrm{pH} 7.25$, containing $115 \mathrm{mM} \mathrm{CsMeSO}_{4}, 20 \mathrm{mM} \mathrm{CsCl}, 10 \mathrm{mM}$ HEPES, $2.5 \mathrm{mM} \mathrm{MgCl}_{2}, 4 \mathrm{mM}$ $\mathrm{Na}_{2} \mathrm{ATP}, 0.4 \mathrm{mM} \mathrm{Na} \mathrm{m}_{3} \mathrm{GP}, 10 \mathrm{Na}$-phosphocreatinine, and 0.6 mM EGTA.
Drug treatment. Systemically administered drugs were given s.c. RU486 (40 $\mu \mathrm{g} / \mathrm{g}, 8 \mu \mathrm{g} / \mathrm{g}$, and $1.6 \mu \mathrm{g} / \mathrm{g}$ of body weight; Sigma-Aldrich) was dissolved in DMSO and injected twice a day during isolation. For local injection of RU486, RU486 was dissolved in 100\% EtOH (final $50 \mathrm{mg} / \mathrm{ml}$ ) and mixed with PBS (RU486/PBS = 1:1,000). $200 \mathrm{nl}$ of virus-containing solution was added to $0.8 \mu \mathrm{l}$ of this mixture and then injected into the barrel cortex. Corticosterone ( $4.17 \mu \mathrm{g} / \mathrm{g}$ or $0.83 \mu \mathrm{g}$ per day; Sigma-Aldrich) was dissolved in sesame oil and injected once a day at P4-P7 or P7-P11. Propranolol (3 $\mu \mathrm{g} / \mathrm{g}$ of body weight; Sigma-Aldrich) was dissolved in saline (62) and injected once a day 30 minutes before isolation.

Corticosterone assay. Plasma corticosterone levels were determined in parallel groups of rats under isolated and nonisolated conditions. Rats were decapitated before isolation or after isolation (in nonisolated groups, decapitation was performed at the same time as the termination of isolation). Trunk blood was collected in a tube and stored on ice. After centrifugation at $3,000 \mathrm{~g}$ for 20 minutes, the supernatant was collected for the assay. Corticosterone plasma concentrations were determined by an enzyme immunoassay kit (DSL) by using 96-well microtiter plates coated with goat anti-rabbit IgG raised against anti-rat corticosterone IgG (DSL). The absorbance levels were measured with a photometric microplate reader (Bio-Rad) at $450 \mathrm{~nm}$. The sensitivity was $1.6 \mathrm{ng} / \mathrm{ml}$, and coefficients of variation within and between assays were $2.3 \%-6.1 \%$.

Immunohistochemistry. SD rats at P11 were used in this experiment. Following heparinized phosphate buffer (PB), perfusion through the cardiac ventricle was done with $4 \%$ paraformaldehyde in $\mathrm{PB}(\mathrm{pH} 7.5)$ at approximately $4^{\circ} \mathrm{C}$. After perfusion, the brains were removed from the cranium, fixed overnight at $4^{\circ} \mathrm{C}$ in $\mathrm{PB}$ containing $4 \%$ paraformaldehyde, and incubated overnight at $4^{\circ} \mathrm{C}$ in $25 \%$ sucrose in $\mathrm{PB}$. The brains were then frozen with powdered dry ice and stored at $-70^{\circ} \mathrm{C}$ until immunohistochemical processing. $30-\mu \mathrm{m}$ frozen coronal sections were cut with a Bright cryostat and washed with $0.1 \mathrm{M}$ PBS. Samples were incubated overnight at $4^{\circ} \mathrm{C}$ with rabbit polyclonal GR antibody diluted 1:2,000 (sc-1004; Santa Cruz Biotechnology Inc.) in PBS containing 1.5\% normal goat serum and 0.1\% Triton X-100. On the next day, sections were incubated with biotinylated anti-rabbit IgG (1:200), followed by incubation with streptavidin-biotin-peroxidase complex (Vectastain Elite ABC Kit; Vectastain Labs). Bound peroxidase was visualized by incubating the sections for 3 minutes in $0.05 \% 3.3^{\prime}$-diaminobenzidine with $\mathrm{H}_{2} \mathrm{O}_{2}$. Samples were then mounted on glass slides, dehydrated in graded alcohol, cleared in xylene, and coverslipped with Permount.

Biochemical analysis. Barrel cortex samples were obtained at specific time points after isolation or under control conditions. After decapitation, tissue samples were put into prechilled tubes and stored at $-80^{\circ} \mathrm{C}$. Nuclear extracts were prepared using an NE-PER kit (Pierce). Synaptosome fractions were prepared as previously described (63). Briefly, samples were homogenized in ice-cold homogenization buffer using glass-glass tissue homogenizer. Homogenates were passed through $2100-\mu \mathrm{m}$-pore nylon mesh filters, then through a $5-\mu \mathrm{m}$-pore filter. Filtered homogenates were centrifuged at $3,600 \mathrm{~g}$ for 10 minutes at $4^{\circ} \mathrm{C}$. Resultant pellets were resuspended in $200 \mu \mathrm{l}$ boiling $1 \%$ SDS, then boiled for 5 minutes. These proteins were separated by SDS-PAGE ( $3 \%-20 \%$ gradient gel; WAKO) and transferred onto PVDF membranes (Millipore). The proteins were detected with the following relevant primary antibodies: (nuclear assay) rabbit polyclonal GR antibody (1:1,000; Santa Cruz Biotechnology Inc.) and cAMP response element-binding protein (CREB) antibody (1:2,000; Upstate), used as a reference for the quantitative analysis. (synaptosome assay), rabbit polyclonal CaMKII and pCaMKII antibodies (1:2,000; Cell Signaling Technology), and rabbit polyclonal p831 and GluR1 (1:1,000; Millipore). The amount of actin (1:5,000; Sigma-Aldrich) was used as a reference for the quantitative analysis of p831. Immunolabeled proteins were detected using LAS3000 (Fujifilm). These data were quantified with ImageQuant software. 
In vivo recording. At $\mathrm{P} 21$, rats were anesthetized with urethane $(1.25 \mathrm{mg} /$ $\mathrm{kg})$. In vivo recordings were performed on several animals in each group $(n=7-10)$. The skull above the barrel cortex was removed as described above. The barrel column was chosen at random for each animal. Wire electrodes were inserted at $200-400 \mu \mathrm{m}$ below the pia. Wire electrodes consisted of 4 approximately $12.5-\mu \mathrm{m}$ (0.0005 inch) tungsten wires (California Fine Wire) bound together by twisting to compose a "tetrode." Successful insertion of electrodes into layer $2 / 3$ was confirmed by histological examination. Single whiskers on the contralateral side were stimulated using a metal needle mounted on a piezoelectric wafer. For each stimulus trial, a whisker was moved up followed by a downward deflection after $0.5 \mathrm{~ms}$. The movement was $0.5 \mathrm{~mm}$ to $1 \mathrm{~mm}$ away from the skin. After amplification and band-pass filtering ( $1 \mathrm{~Hz}-5 \mathrm{kHz}$; model 4 channels), field potentials and action potentials were recorded continuously using Digital Lynx (Neuralynx Inc). Recording sessions lasted from 30 to 60 minutes, and then the data were analyzed off-line. Wide-band continuous brain signals were highpass filtered and thresholded to detect action potentials. The extracted spike waveforms were separated on the basis of their spike amplitude and wave shape using principal component analysis (PCA). A single spike was represented by 12 waveform parameters as a 12 -dimensional feature vector. Units were identified and isolated by an automatic clustering program (KlustaKwik Program). Extraction of single-unit activity is demonstrated in Supplemental Figure 4, A-C. The number of spikes calculated from 5 to $50 \mathrm{~ms}$ after whisker deflection was counted and used as a measure of the response. The slopes of the initial negative deflection of field responses were computed by a custom-made computer program.

Gap-crossing test. The apparatus used in the modified version of the gapcrossing-task is shown in Figure 6A. The platform was 1 meter from the ground. To ensure unbiased evaluation, experimenters were blinded to animal groups during analysis. One day before testing, rats were food deprived for 24 hours. Twelve hours before testing, both eyes were sutured. Rats were initially trained to find a reward (chow pellet) at the opposite end of a runway. After this training phase, a gap was inserted in the runway and was widened at $0.5-\mathrm{cm}$ intervals. If rats crossed the gap to obtain a food reward within 2 minutes, it was considered a successful trial. The gap was widened until rats would no longer step across it (within the 2-minute period). This gap size was defined as SU. Subsequently, the gap was narrowed at $0.5-\mathrm{cm}$ intervals until rats crossed it. This gap size was defined as SL. The difference between SU and SL was calculated. This protocol was repeated 5 times in succession to obtain an average $\Delta S$ value. The animals were able to obtain a food reward for each trial. This value reflects the ability of distance discrimination and is dependent on whisker-barrel function, since animals with disrupted whisker-barrel mapping by the expression of GFP-GluR1ct in layer $2 / 3$ of barrel cortex showed larger $\Delta$ S (Figure $6 \mathrm{~A}$ ). To determine general anxiety, we assessed movements of rats such as distance traveled just after food deprivation.
Forelimb placing test. To ensure unbiased evaluation, experimenters were blinded to animal groups during analysis. The rat's torso was supported by the experimenter and suspended such that all 4 legs hung freely in the air. The experimenter then brought the rat toward the edge of a tabletop, taking care to avoid abrupt movements that might trigger placing due to a vestibular response. If such responses were noted, they were extinguished by taking the rat through the testing motions in open space (i.e., away from the tabletop) a few times. The rat's vibrissae were brushed against the table edge on the same side of the body in which forelimb placing was evaluated. The percentage of 10 trials in which the rat successfully placed its forepaw onto the tabletop was recorded for each side.

Statistics. All data are presented as mean \pm SEM. Data were analyzed by paired, 2-tailed $t$ test or 1-way factorial ANOVA. In Supplemental Figures, data were analyzed by paired, 2-tailed $t$ test, except for that in Supplemental Figures 5 and 6 , which was analyzed by 1 -way factorial ANOVA. ANOVA was followed by post-hoc analysis with the Fisher protected least-significant difference test. $P<0.05$ was considered statistically significant.

Study approval. All animal experiments were approved by the Institutional Animal Care and Use Committee of the Animal Research Center, Yokohama City University Graduate School of Medicine and Cold Spring Harbor Laboratory.

\section{Acknowledgments}

This project was supported by Grant-in-Aid for Young Scientists (Start-up) (18800037), Grant-in-Aid for Scientific Research (20300131) (to T. Takahashi), JST, CREST (to T. Takahashi), Special Coordination Funds for Promoting Science and Technology (to T. Takahashi), the Sumitomo Foundation (to T. Takahashi), the KANAE Foundation (to T. Takahashi), the Takeda Science Foundation (to T. Takahashi), NARSAD (to T. Takahashi), the grant for 2007 Strategic Research Project (K19025) of Yokohama City University (to T. Takahashi), "Development of biomarker candidates for social behavior" carried out under the Strategic Research Program for Brain Sciences by the Ministry of Education, Culture, Sports, Science and Technology of Japan (to T. Takahashi), and the NIH (MH049159 to R. Malinow). We also thank Yoshiko Kannno for excellent technical assistance and Helmut Kessels for the critical reading of the manuscript.

Received for publication January 26, 2012, and accepted in revised form May 9, 2012.

Address correspondence to: Takuya Takahashi, Yokohama City University, Graduate School of Medicine, 3-9 Fukuura, Kanazawa-ku, Yokohama 236-0004, Japan. Phone: 81.0.45.787.2579; Fax: 81.0.45.787.2578; E-mail: takahast@yokohama-cu.ac.jp.
1. Hu $\mathrm{H}$, et al. Emotion enhances learning via norepinephrine regulation of AMPA-receptor trafficking. Cell. 2007;131(1):160-173.

2. Enthoven L, Oitzl MS, Koning N, van der Mark M, de Kloet ER. Hypothalamic-pituitary-adrenal axis activity of newborn mice rapidly desensitizes to repeated maternal absence but becomes highly responsive to novelty. Endocrinology. 2008;149(12):6366-6377.

3. McEwen BS. Protective and damaging effects of stress mediators: central role of the brain. Dialogues Clin Neurosci. 2006;8(4):367-381.

4. Insel TR, Fernald RD. How the brain processes social information: searching for the social brain. Annu Rev Neurosci. 2004;27:697-722.

5. Khonicheva NM, Loseva EV, Chabak-Garbach R, Loriya MV, Airapetyants MG. A special case of learning disorder in isolant rats as a model of disintegra- tion. Neurosci Behav Physiol. 2006;36(6):597-603.

6. Stranahan AM, Khalil D, Gould E. Social isolation delays the positive effects of running on adult neurogenesis. Nat Neurosci. 2006;9(4):526-533.

7. Wallace DL, et al. CREB regulation of nucleus accumbens excitability mediates social isolation-induced behavioral deficits. Nat Neurosci. 2009;12(2):200-209.

8. Fox K. Anatomical pathways and molecular mechanisms for plasticity in the barrel cortex. Neuroscience. 2002;111(4):799-814.

9. Buonomano DV, Merzenich MM. Cortical plasticity: from synapses to maps. Annu Rev Neurosci. 1998;21:149-186.

10. Ahl AS. The role of vibrissae in behavior: a status review. Vet Res Commun. 1986;10(4):245-268.

11. Strozik E, Festing MF. Whisker trimming in mice. Lab Anim. 1981;15(4):309-312.
12. Takahashi T, Svoboda K, Malinow R. Experience strengthening transmission by driving AMPA receptors into synapses. Science. 2003; 299(5612):1585-1588.

13. Miyazaki T, et al. Developmental AMPA receptor subunit specificity during experience-driven synaptic plasticity in the rat barrel cortex. Brain Res. 2012;1435:1-7.

14. Bredt DS, Nicoll RA. AMPA receptor trafficking at excitatory synapses. Neuron. 2003;40(2):361-379.

15. Hollmann M, Heinemann S. Cloned glutamate receptors. Annu Rev Neurosci. 1994;17:31-108.

16. Barry MF, Ziff EB. Receptor trafficking and the plasticity of excitatory synapses. Curr Opin Neurobiol. 2002;12(3):279-286.

17. Malinow R, Malenka RC. AMPA receptor trafficking and synaptic plasticity. Annu Rev Neurosci. 
2002;25:103-126.

18. Scannevin RH, Huganir RL. Postsynaptic organization and regulation of excitatory synapses. Nat Rev Neurosci. 2000;1(2):133-141.

19. Hayashi Y, Shi SH, Esteban JA, Piccini A, Poncer JC, Malinow R. Driving AMPA receptors into synapses by LTP and CaMKII: requirement for GluR1 and PDZ domain interaction. Science. 2000; 287(5461):2262-2267.

20. Plant K, et al. Transient incorporation of native GluR2-lacking AMPA receptors during hippocampal long-term potentiation. Nat Neurosci. 2006; 9(5):602-604.

21. Clem RL, Barth A. Pathway-specific trafficking of native AMPARs by in vivo experience. Neuron. 2006;49(5):663-670.

22. Jitsuki S, et al. Serotonin mediates cross-modal reorganization of cortical circuits. Neuron. 2011; 69(4):780-792.

23. Rumpel S, LeDoux J, Zador A, Malinow R. Postsynaptic receptor trafficking underlying a form of associative learning. Science. 2005;308(5718):83-88.

24. Mitsushima D, Ishihara K, Sano A, Kessels HW, Takahashi T. Contextual learning requires synaptic AMPA receptor delivery in the hippocampus. Proc Natl Acad Sci U S A. 2011;108(30):12503-12508.

25. Kessels HW, Malinow R. Synaptic AMPA receptor plasticity and behavior. Neuron. 2009;61(3):340-350

26. Engblom D, et al. Glutamate receptors on dopamine neurons control the persistence of cocaine seeking. Neuron. 2008;59(3):497-508.

27. Lee HK, et al. Phosphorylation of the AMPA receptor GluR1 subunit is required for synaptic plasticity and retention of spatial memory. Cell. 2003; 112(5):631-643.

28. Reisel D, et al. Spatial memory dissociations in mice lacking GluR1. Nat Neurosci. 2002;5(9):868-873.

29. Kehoe P, Bronzino JD. Neonatal stress alters LTP in freely moving male and female adult rats. Hippocampus. 1999;9(6):651-658.

30. Kim JJ, Diamond DM. The stressed hippocampus, synaptic plasticity and lost memories. Nat Rev Neu rosci. 2002;3(6):453-462.

31. Diamond DM, Fleshner M, Rose GM. Psychological stress repeatedly blocks hippocampal primed burst potentiation in behaving rats. Behav Brain Res. 1994;62(1):1-9.

32. Diamond DM, Park CR. Predator exposure produces retrograde amnesia and blocks synaptic plasticity. Progress toward understanding how the hippocampus is affected by stress. Ann NY Acad Sci. 2000; 911:453-455.

33. Foy MR, Stanton ME, Levine S, Thompson RF. Behavioral stress impairs long-term potentiation in rodent hippocampus. Behav Neural Biol. 1987;48(1):138-149.
34. Etherton M, et al. Autism-linked neuroligin-3 R451C mutation differentially alters hippocampal and cortical synaptic function. Proc Natl Acad Sci US A. 2011;108(33):13764-13769.

35. Diamond ME, Huang W, Ebner FF. Laminar comparison of somatosensory cortical plasticity. Science. 1994;265(5180):1885-1888.

36. McGowan PO, et al. Epigenetic regulation of the glucocorticoid receptor in human brain associates with childhood abuse. Nat Neurosci. 2009;12(3):342-348.

37. Stern EA, Maravall M, Svoboda K. Rapid development and plasticity of layer $2 / 3$ maps in rat barrel cortex in vivo. Neuron. 2001;31(2):305-315.

38. Zimmerberg B, Rosenthal AJ, Stark AC. Neonatal social isolation alters both maternal and pup behaviors in rats. Dev Psychobiol. 2003;42(1):52-63.

39. Kessels HW, Kopec CD, Klein ME, Malinow R. Roles of stargazin and phosphorylation in the control of AMPA receptor subcellular distribution. Nat Neurosci. 2009;12(7):888-896.

40. Lisman J, Schulman H, Cline H. The molecular basis of CaMKII function in synaptic and behavioural memory. Nat Rev Neurosci. 2002;3(3):175-190.

41. Derkach VA, Oh MC, Guire ES, Soderling TR. Regulatory mechanisms of AMPA receptors in synaptic plasticity. Nat Rev Neurosci. 2007;8(2):101-113.

42. Hazan L, Zugaro M, Buzsaki G. Klusters, NeuroScope, NDManager: a free software suite for neurophysiological data processing and visualization. J Neurosci Methods. 2006;155(2):207-216.

43. Barneoud P, Gyger M, Andres F, van der Loos H. Vibrissa-related behavior in mice: transient effect of ablation of the barrel cortex. Behav Brain Res. 1991; 44(1):87-99.

44. Hutson KA, Masterton RB. The sensory contribution of a single vibrissa's cortical barrel. J Neurophysiol. 1986;56(4):1196-1223.

45. Felt BT, Schallert T, Shao J, Liu Y, Li X, Barks JD. Early appearance of functional deficits after neonatal excitotoxic and hypoxic-ischemic injury: fragile recovery after development and role of the NMDA receptor. Dev Neurosci. 2002;24(5):418-425.

46. Feder A, Nestler EJ, Charney DS. Psychobiology and molecular genetics of resilience. Nat Rev Neurosci. 2009;10(6):446-457.

47. Roozendaal B, McEwen BS, Chattarji S. Stress, memory and the amygdala. Nat Rev Neurosci. 2009; 10(6):423-433.

48. Groc L, Choquet D, Chaouloff F. The stress hormone corticosterone conditions AMPAR surface trafficking and synaptic potentiation. Nat Neurosci. 2008;11(8):868-870.

49. Gerges NZ, Aleisa AM, Schwarz LA, Alkadhi KA. Reduced basal CaMKII levels in hippocampal CA1 region: possible cause of stress-induced impair- ment of LTP in chronically stressed rats. Hippocampus. 2004;14(3):402-410.

50. Harlow EG, Till SM, Russell TA, Wijetunge LS, Kind $\mathrm{P}$, Contractor A. Critical period plasticity is disrupted in the barrel cortex of FMR1 knockout mice. Neuron. 2010;65(3):385-398.

51. Marco EJ, Hinkley LB, Hill SS, Nagarajan SS. Sensory processing in autism: a review of neurophysiologic findings. Pediatr Res. 2011;69(5 pt 2):48R-54R.

52. Leitman DI, Sehatpour P, Higgins BA, Foxe JJ, Silipo G, Javitt DC. Sensory deficits and distributed hierarchical dysfunction in schizophrenia. Am J Psychiatry. 2010;167(7):818-827.

53. Magerl W, Burkart D, Fernandez A, Schmidt LG, Treede RD. Persistent antinociception through repeated self-injury in patients with borderline personality disorder. Pain. 2012;153(3):575-584.

54. Hendler T, Rotshtein P, Hadar U. Emotion-perception interplay in the visual cortex: "the eyes follow the heart”. Cell Mol Neurobiol. 2001;21(6):733-752.

55. Pitman RK, Orr SP, Shalev AY, Metzger LJ, Mellman TA. Psychophysiological alterations in posttraumatic stress disorder. Semin Clin Neuropsychiatry. 1999;4(4):234-241.

56. Hendler T, et al. Sensing the invisible: differential sensitivity of visual cortex and amygdala to traumatic context. Neuroimage. 2003;19(3):587-600.

57. Schmahl C, et al. Differential nociceptive deficits in patients with borderline personality disorder and self-injurious behavior: laser-evoked potentials, spatial discrimination of noxious stimuli, and pain ratings. Pain. 2004;110(1-2):470-479.

58. Miller LJ, Nielsen DM, Schoen SA, Brett-Green BA. Perspectives on sensory processing disorder: a call for translational research. Front Integr Neurosci. 2009;3:22.

59. Kolleker A, et al. Glutamatergic plasticity by synaptic delivery of GluR-B(long)-containing AMPA receptors. Neuron. 2003;40(6):1199-1212.

60. Zhu JJ, Esteban JA, Hayashi Y, Malinow R. Postnatal synaptic potentiation: delivery of GluR4-containing AMPA receptors by spontaneous activity. Nat Neurosci. 2000;3(11):1098-1106.

61. Lois C, Hong EJ, Pease S, Brown EJ, Baltimore D. Germline transmission and tissue-specific expression of transgenes delivered by lentiviral vectors. Science. 2002;295(5556):868-872.

62. Roozendaal B, Okuda S, Van der Zee EA, McGaugh JL. Glucocorticoid enhancement of memory requires arousal-induced noradrenergic activation in the basolateral amygdala. Proc Natl Acad Sci US A. 2006;103(17):6741-6746.

63. Whitlock JR, Heynen AJ, Shuler MG, Bear MF. Learning induces long-term potentiation in the hippocampus. Science. 2006;313(5790):1093-1097. 